\title{
Vortex Flow and Transverse Flux Screening at the Bose Glass Transition
}

\author{
A. W. Smith, ${ }^{1}$ H. M. Jaeger, ${ }^{1}$ T. F. Rosenbaum, ${ }^{1}$ A. M. Petrean, ${ }^{2}$ W. K. Kwok,${ }^{2}$ and G. W. Crabtree ${ }^{2}$ \\ ${ }^{1}$ The James Franck Institute and Department of Physics, The University of Chicago, Chicago, Illinois 60637 \\ ${ }^{2}$ Materials Science Division, Argonne National Laboratory, Argonne, Illinois 60439
}

(Received 3 December 1999)

\begin{abstract}
We study the vortex phase diagram in untwinned YBCO crystals with columnar defects. These randomly distributed defects are expected to induce a "Bose glass" phase of localized vortices exhibiting a vanishing resistance and Meissner effect for magnetic fields $H_{\perp}$ transverse to the columns. We directly observe the transverse Meissner effect using a Hall probe array. As predicted, the Meissner state breaks down at temperatures $T_{s}$ that decrease linearly as $H_{\perp}$ increases. However, $T_{s}$ lies far below the conventional melting temperature $T_{m}$ determined by a vanishing resistivity, suggesting a regime where vortices are effectively localized even when rotated off the columnar defects.
\end{abstract}

PACS numbers: $74.60 . \mathrm{Ge}, 74.25 . \mathrm{Bt}$

Weak pinning, large anisotropy, and prodigious thermal fluctuations in high temperature superconductors permit magnetic vortices to exist in a liquid phase which flows easily in response to an external current [1]. This technologically undesirable state of finite electrical resistance is quelled only at sufficiently low temperatures when vortex-vortex interactions precipitate a solid phase [2-4]. Columnar defects, a form of strong disorder correlated across many $\mathrm{CuO}_{2}$ planes, serve as an effective strategy to bolster the constraining effects of the interaction potential, localizing the vortices on the disordered array of columns. The resulting "Bose glass" phase [5] exhibits two characteristic signatures: a diverging vortex viscosity leading to a zero resistance state, as well as a diverging tilt modulus resulting in vortex alignment with the columns even as the external magnetic field rotates. A large rotation of the external field, however, will melt the glass at fixed temperature. We monitor both the onset of a resistive response (vortex mobility) and the onset of transverse flux penetration (vortex alignment). In contrast to theory, we find that these two events yield widely different demarcations for the Bose glass phase.

Amorphous columnar tracks created by heavy ion bombardment of cuprate superconductors are of nearly optimal size and geometry to pin vortices over their entire length. Thermal excitations, however, permit some segment of a vortex line to wander off its pinning site. Nelson and Vinokur [5] have mapped this statistical mechanics problem onto the quantum mechanical problem of two-dimensional bosons in the presence of point disorder [6]. They find a sharp phase transition between a high-temperature vortex liquid of delocalized and entangled vortex lines and a low temperature Bose glass dominated by the spatial disorder in the columnar defect distribution. The predictions of scaling theory [5,7-9] have been tested [10-19] by measuring the vortex mobility subjected to a driving current at the approach to the glass transition temperature, $T_{\mathrm{BG}}$. These experiments vary widely on values for the critical exponents and on the angular dependence of $T_{\mathrm{BG}}$. We consider an additional prediction of the theory, that below
$T_{\mathrm{BG}}(\vec{H})$ vortices remain parallel to the columnar defects even when the external magnetic field is rotated off the defect axis. While this is a striking element of the theory, it has so far been untapped as a quantitative probe of the nature of the glass transition [20].

With our experimental configuration, illustrated in Fig. 1, it is possible to measure both the vortex mobility and the extent to which flux lines remain parallel to the columnar defects. We sense the vortex mobility using an 8 turn, $0.3 \mathrm{~mm}$ diameter pickup coil near the sample. Since each vortex carries a fixed amount of magnetic flux, changes in the vortex density appear as an induced voltage in the pickup coil. In the presence of a small ac driving field this voltage signal provides a measure of how easily vortices hop from columnar defect to columnar defect. A micron-sized InAs Hall probe array [21] under the sample measures the local magnetic field component perpendicular to the columnar defects. This provides a direct gauge of vortex rotation in response to the transverse component of the applied field. The $\mathrm{YBa}_{2} \mathrm{Cu}_{3} \mathrm{O}_{7-\delta}(\mathrm{YBCO})$ untwinned single crystal with dimensions $0.5 \times 0.5 \times 0.035 \mathrm{~mm}^{3}$, critical temperature $T_{c}=92.5 \mathrm{~K}$, and transition width $\Delta T_{c}=0.3 \mathrm{~K}$ was irradiated with $4 \mathrm{GeV} \mathrm{Au}$ ions at the National Superconducting Cyclotron Laboratory, producing columnar defects parallel to the $c$ axis with equivalent vortex density $B_{\Phi}=0.5 \mathrm{~T}$.

We cooled the $\mathrm{YBa}_{2} \mathrm{Cu}_{3} \mathrm{O}_{7-\delta}$ single crystal from the normal state at a fixed parallel magnetic field $H_{\|}$and then ramped up a perpendicular field $H_{\perp}$. The parallel component of the field was aligned carefully with the columnar defects using a 3-axis split-bore magnet system, with the magnetic field direction adjusted to minimize the vortex mobility determined by the pickup coil. The Hall probes recorded the local perpendicular magnetic field as $H_{\perp}$ was swept through cycles $H_{\perp}^{\max } \rightarrow-H_{\perp}^{\max } \rightarrow H_{\perp}^{\max }$ with successively increasing ramp end points $\pm H_{\perp}^{\max }$. The local field underneath the crystal remained substantially reduced from that on either side at small $H_{\perp}$ (Fig. 1), indicating a $30 \%$ diamagnetic shielding of the external field [22]. This behavior is independent of the direction of the field ramp, 


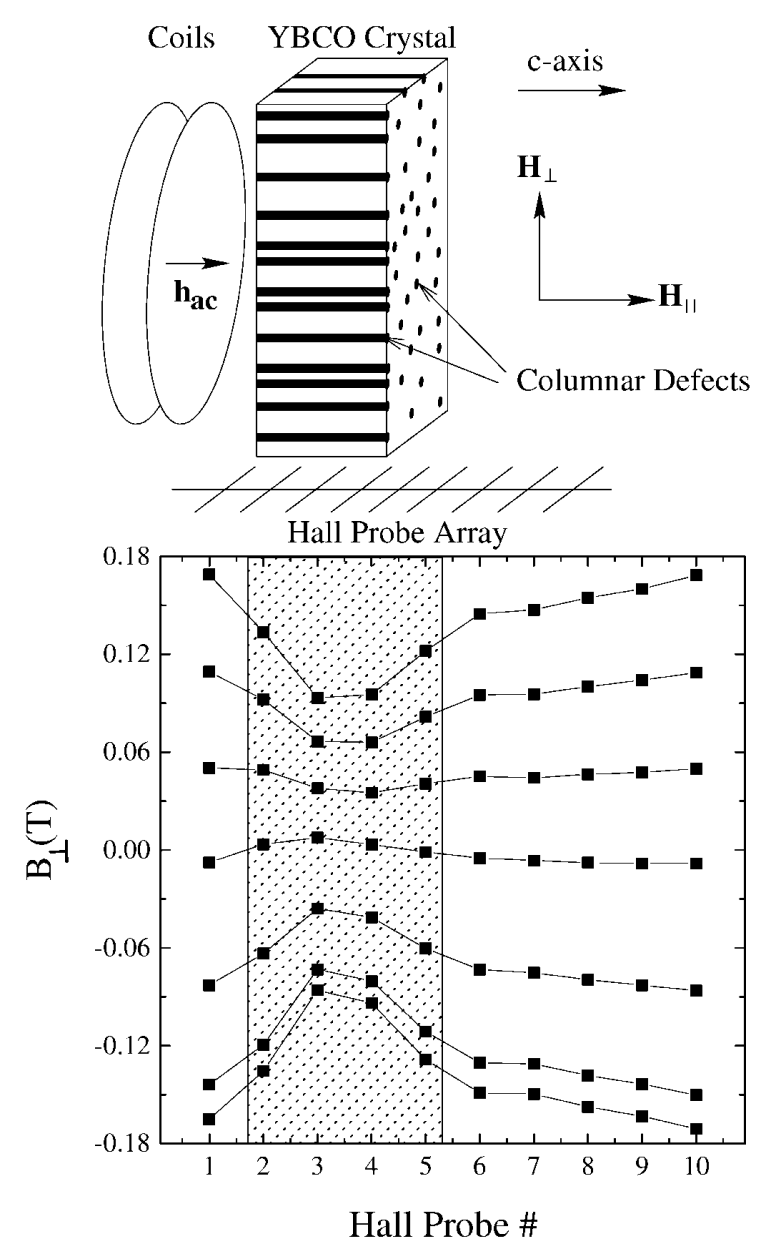

FIG. 1. Top: Sketch of the experimental setup. A superconducting solenoid and two shimming coils generate a magnetic field $H_{\|}$parallel and $H_{\perp}$ transverse to the columnar defects, respectively. Close to the sample a pickup coil detects vortex motion in response to a small ac driving field $h_{\mathrm{ac}}$ at $1000 \mathrm{~Hz}$ and less than $0.2 \mathrm{G}$. The $\mathrm{YBa}_{2} \mathrm{Cu}_{3} \mathrm{O}_{7-\delta}$ crystal rests on its edge atop a ten element linear array of Hall probes. The probes are spaced $10 \mu \mathrm{m}$ apart with active area $3 \times 3 \mu \mathrm{m}^{2}$ and positioned to monitor the magnetic field component $B_{\perp}$ transverse to the columnar defects both directly underneath the crystal and far away from its edges. Bottom: $B_{\perp}$ vs Hall probe number as $H_{\perp}$ is ramped from positive to negative values. The data were taken at temperature $T=10 \mathrm{~K}$ and parallel magnetic field $H_{\|}=0.4 B_{\Phi}$. Data points connected by lines were measured simultaneously. The YBCO crystal (shaded region) is centered between Hall probes 3 and 4 . Underneath the sample $\left|B_{\perp}\right|$ is always smaller than that farther away, indicating diamagnetic screening and a transverse Meissner effect in the Bose glass state.

ruling out an alternative explanation of vortex penetration in a Bean profile. At sufficiently large $H_{\perp}$, hysteresis sets in, the field profile acquires a component that depends on ramp direction, and the vortices no longer remain aligned with the columns. The perpendicular magnetization $M_{\perp}$ adds further support to this scenario [23]. We find that for sufficiently small $H_{\perp}, M_{\perp} \propto-H_{\perp}$. Moreover, there is negligible hysteresis indicating that the superconductor expels the component of applied field perpendicular to the columnar defects. However, at sufficiently large $H_{\perp}, M_{\perp}$ becomes hysteretic and is no longer proportional to $-H_{\perp}$.
We quantify this breakdown of the transverse Meissner effect in terms of a critical tilt angle $\theta_{c}=\tan ^{-1}\left(H_{\perp}^{c} / H_{\|}\right)$. In order to determine $H_{\perp}^{c}$, the field at which transverse flux enters the superconductor, we measured the hysteresis in the local field under the sample $B_{\perp}$ as a function of $H_{\perp}^{\max }$. We plot in the inset in Fig. 2 the hysteresis width of the magnetization loops $B_{\perp}\left(H_{\perp}\right)$. This width remains negligible until $H_{\perp}$ reaches a critical value $H_{\perp}^{c}$, beyond which it increases sharply. We have checked explicitly that the value for $H_{\perp}^{c}$ is independent of position underneath the crystal; it is the same for Hall probes near the sample center (No. 4) and near the sample edge (No. 6). By contrast, the hysteresis width of an unirradiated YBCO crystal at the same temperature and parallel magnetic field shows no such offset in $H_{\perp}$.

Our direct observation of a transverse Meissner effect validates a central prediction of the Bose glass theory. Moreover, from measurements of the critical transverse field $H_{\perp}^{c}(T)$ over a range of temperatures we can define a maximum screening temperature $T_{s}\left(H_{\perp}\right)$ above which the transverse Meissner effect breaks down. The main panel in Fig. 2 shows $T_{s}$ as a function of tilt angle. With increasing $B_{\|}$the ability to screen transverse flux diminishes and smaller tilt angles are required to significantly suppress $T_{s}$. Specifically, for applied fields $H=0.4 B_{\Phi}$ and $1.0 B_{\Phi}$ our data over a range of more than $40 \mathrm{~K}$ exhibit a linear decrease of $T_{s}$ with $\theta$. Here $B_{\Phi}$ is the matching field at which the vortex density equals the density of columnar defects

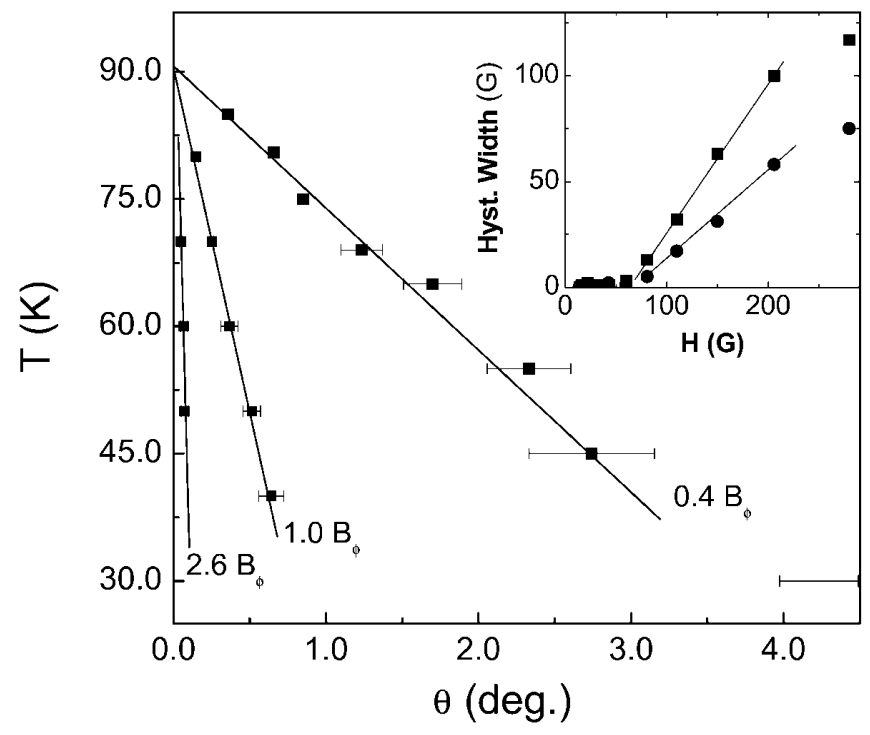

FIG. 2. Main panel: Screening temperature $T_{s}$ vs perpendicular magnetic field $H_{\perp}$ for the indicated values of $H_{\|}$, the magnetic field parallel to the columnar defects (note $B_{\Phi}=0.5 \mathrm{~T}$ ). Inset: Hysteresis width vs amplitude of field ramp $H_{\perp}^{\max }$ for Hall probes 4 and 6 measured at $T=65 \mathrm{~K}$ and $H_{\|}=0.4 B_{\Phi}$. The hysteresis width was determined from plots of $B_{\perp}\left(H_{\perp}\right)$ and was taken as the difference between the values of $H_{\perp}^{*}$ for which $B_{\perp}\left(H_{\perp}^{*}=0\right)$ as $H_{\perp}$ was ramped through zero from above and below. The hysteresis in both Hall probes remains negligible up to a threshold field $H_{\perp}^{c}$, which we identify with the breakdown of transverse flux screening. 
[24]. Once $H_{\|}>B_{\Phi}$ screening appears to become rapidly ineffective; at $H_{\|}=2.6 B_{\Phi}, T_{s}$ has been suppressed by $50 \%$ within the first $0.1 \mathrm{deg}$ of tilt.

We now compare these results for the transverse Meissner effect with measurements of the onset of vortex mobility. Using the pickup coil shown in Fig. 1, we tracked the locus of temperature and magnetic field data for which the linear resistivity $\rho(T)$ drops below a fixed threshold (taken here as $1 / 1000$ of the normal state resistivity at current density $j \simeq 10 \mathrm{~A} / \mathrm{cm}^{2}$ ). Given the strong temperature dependence of $\rho(T)$ as the zero-resistance state is approached, this type of resistance threshold criterion conventionally has been used to monitor the melting temperature $T_{m}$. The normalized change in the melting temperature, $\Delta T_{m} / T_{m}=\left[T_{m}\left(H_{\perp}=0\right)-T_{m}\left(H_{\perp}\right)\right] / T_{m}$, reveals a cusp over a broad range of tilt angles $\theta$ (Fig. $3 \mathrm{a}$ ). By comparison to the screening temperature $T_{s}$ in Fig. 2, the melting temperature $T_{m}$ varies nonlinearly and only weakly with $H_{\perp}$.

If we associate the Bose glass temperature $T_{\mathrm{BG}}\left(H_{\perp}\right)$ with the demise of the transverse Meissner effect by setting $T_{s}\left(H_{\perp}\right)=T_{\mathrm{BG}}\left(H_{\perp}\right)$, we can determine the static critical exponent $\nu$ for the glass transition via the relation [9] $T_{\mathrm{BG}}\left(H_{\perp}=0\right)-T_{\mathrm{BG}}\left(H_{\perp}\right) \propto H_{\perp}^{1 / \nu}$. From the data for
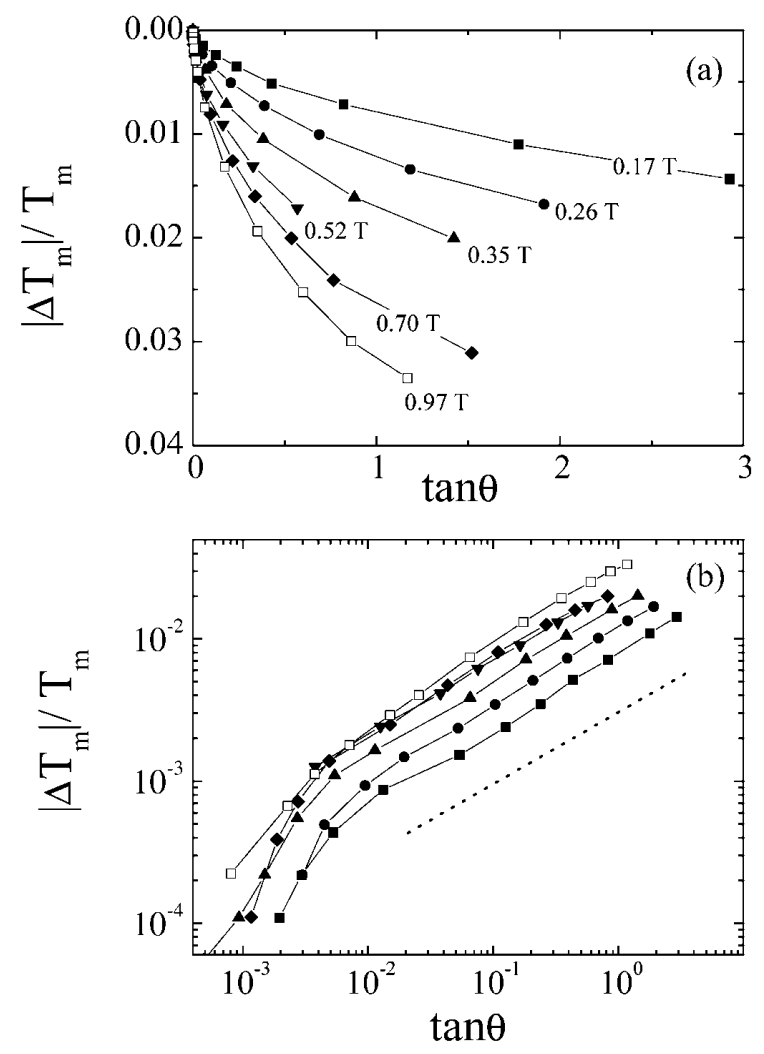

FIG. 3. (a) Relative change in vortex melting temperature $\Delta T_{m} / T_{m}=\left[T_{m}\left(H_{\perp}=0\right)-T_{m}\left(H_{\perp}\right)\right] / T_{m}$ vs $\tan \theta$ for the indicated values of magnetic field parallel $H_{\|}$to the columnar defects. (b) Log-log plot showing power law dependence of $\Delta T_{m} \propto H_{\perp}^{1 / \nu}$. The straight line is a guide to the eye with $\nu=2$.
$H_{\|}=0.4 B_{\Phi}$ and $1.0 B_{\Phi}$ (Fig. 2), we find $\nu=0.9 \pm 0.1$ and $1.0 \pm 0.1$, respectively. This result is at the bound $[25,26] \nu=2 / d=1$, with effective system dimensionality $d=2$, and it agrees with computer simulations [9] of Bose glass melting. Our data indicate that even well above $B_{\Phi}$ the exponent $\nu$ remains approximately 1 . As shown in Fig. 3b, $\Delta T_{m} / T_{m}$ over two decades follows a power law in $\tan \theta$ or, equivalently, in $H_{\perp}$. Taking the melting temperature $T_{m}$ as the Bose glass temperature would lead to a critical exponent $\nu=1.9 \pm 0.2$ for $\theta>1^{\circ}$, but with rounding of the cusp at the smallest tilt angles [27]. This value of $\nu$ is consistent with that reported in Ref. [28] where $T_{m}\left(H_{\perp}\right)$ was determined from transport measurements.

The differences between $T_{m}$ and $T_{s}$ also lead to a completely different demarcation between the glass and liquid phases. We combine in Fig. 4 the phase diagrams based on vortex flow and transverse field screening for $H_{\|}=$ $0.4 B_{\Phi}$. The boundary between glass and liquid coincides for both types of measurements at $H_{\perp}=0$, but $T_{m}$ and $T_{s}$ quickly bifurcate with increasing $H_{\perp}$. Not only is the transverse screening limited to a far narrower range of magnetic field tilt angle, it also indicates a Bose glass transition at temperatures many tens of Kelvin below the disappearance of the linear resistive response.

We are left with the intriguing scenario where a new, intermediate regime exists between the glass and the liquid state which exhibits no vortex flow even in the absence of a fully developed transverse Meissner effect. This corresponds to immobilized vortices which are at least

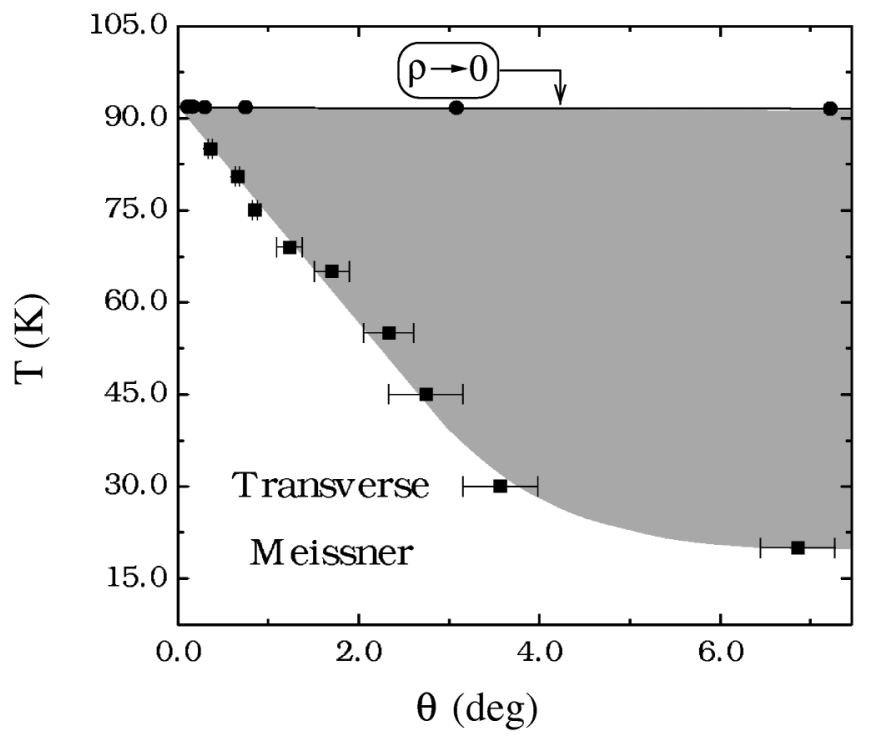

FIG. 4. Combined phase diagram showing both melting (filled circles) and screening (filled squares) temperatures vs tilt angle $\theta$ for $H_{\|}=0.2 \mathrm{~T}$. The two curves coincide at $\theta=0$, but diverge at larger angles. Above the line indicated by the screening temperature, the transverse Meissner effect is lost. Vortex flow, however, does not set in until the melting line is crossed. On this temperature scale the cusp in $\Delta T_{m} / T_{m}$ apparent in Fig. 3 is no longer visible. At sufficiently large tilt angles the fast linear suppression of the screening temperature appears to flatten off. 
partly rotated off the columnar defects. (By contrast, in a highly anisotropic superconductor such as $\mathrm{Bi}_{2} \mathrm{Sr}_{2} \mathrm{CaCu}_{2} \mathrm{O}_{8}$ Josephson vortices may exist independently of immobilized longitudinal vortices [29].) The origin and detailed physical nature of this intermediate regime between $T_{m}$ and $T_{s}$ remain open questions. Our results appear to be in conflict with the current theoretical framework for twodimensional bosonic states which allows for only a single transition from insulating to superconducting behavior. However, there has been mounting experimental evidence in different systems [30] that an intermediate regime may indeed exist. Comparative studies of high- $T_{c}$ superconductors with different types of disorder and different degrees of anisotropy, as well as characterization of the full nonlinear spectral response, should help illuminate this unusual vortex state.

We thank Dr. Reggie Ronningen for assisting with the ion irradiation at the National Superconducting Cyclotron Laboratory. This work was supported by the National Science Foundation through the Science and Technology Center for Superconductivity (DMR91-20000) and under Contract No. PHY-9528844, and by the U.S. Department of Energy, BES-Materials Science under Contract No. W-31-109-ENG-38 (W. K. K., A. M. P., G. W. C.).

[1] D. A. Huse, M. P. A. Fisher, and D. S. Fisher, Nature (London) 368, 553 (1992).

[2] P. L. Gammel, L. F. Schneemeyer, J. V. Waszczak, and D. J. Bishop, Phys. Rev. Lett. 61, 1666 (1988).

[3] A. Schilling et al., Nature (London) 382, 791 (1996).

[4] Charles Reichhardt, Anne van Otterlo, and Gergely T. Zimányi, Phys. Rev. Lett. 84, 1994 (2000).

[5] D. R. Nelson and V. M. Vinokur, Phys. Rev. B 48, 13060 (1993).

[6] M.P. A. Fisher, P. B. Weichman, G. Grinstein, and D.S. Fisher, Phys. Rev. B 40, 546 (1989).

[7] M. Wallin and S. Girvin, Phys. Rev. B 47, 14642 (1993).

[8] D. R. Nelson and L. Radzihovsky, Phys. Rev. B 54, R6845 (1996).

[9] J. Lidmar and M. Wallin, Europhys. Lett. 47, 494 (1999).
[10] L. Civale et al., Phys. Rev. Lett. 67, 648 (1991).

[11] W. Jiang et al., Phys. Rev. Lett. 72, 550 (1994).

[12] L. Krusin-Elbaum et al., Phys. Rev. Lett. 72, 1914 (1994).

[13] R. C. Budhani, W. L. Holstein, and M. Suenaga, Phys. Rev. Lett. 72, 566 (1994).

[14] D. S. Reed, N.-C. Yeh, M. Konczykowski, A. V. Samoilov, and F. Holtzberg, Phys. Rev. B 51, 16448 (1995).

[15] C. J. van der Beek et al., Phys. Rev. Lett. 74, 1214 (1995).

[16] G. Nakielski et al., Phys. Rev. Lett. 76, 2567 (1996).

[17] M. Oussena et al., Phys. Rev. Lett. 76, 2559 (1996).

[18] A. Mazilu et al., Phys. Rev. B 58, R8909 (1998).

[19] S. A. Grigera et al., Phys. Rev. Lett. 81, 2348 (1998).

[20] I. M. Obaidat, S. J. Park, H. Safar, and J. S. Kouvel, Phys. Rev. B 56, R5774 (1997).

[21] E. Pugel, E. Shung, T. F. Rosenbaum, and S. P. Watkins, Appl. Phys. Lett. 71, 2205 (1997).

[22] We do not expect the field under the sample to remain unchanged during the field ramp even when screening is perfect because there is always a nonzero distance between the Hall probe array and the sample.

[23] A. W. Smith, H. M. Jaeger, T. F. Rosenbaum, W. K. Kwok, and G. W. Crabtree (to be published).

[24] A. W. Smith, H. M. Jaeger, T. F. Rosenbaum, W. K. Kwok, and G. W. Crabtree, Phys. Rev. B 59, R11 665 (1999).

[25] J. T. Chayes, L. Chayes, D. S. Fisher, and T. Spencer, Phys. Rev. Lett. 57, 2999 (1986).

[26] F. Pázmándi, R. T. Scalettar, and G. T. Zimányi, Phys. Rev. Lett. 79, 5130 (1997).

[27] Taking the recent correction (Refs. [7,9]) to the scaling function into account, we note that previous experimental determinations of $\nu$ from vortex flow measurements in YBCO samples have found values of $\nu$ ranging from 1 to 3 (Refs. [11,14,16,19,20]). Our vortex flow data range extends to much smaller tilt angles, and within the scatter the data for $\theta<1^{\circ}$ are consistent with $\nu \simeq 1$. Whether $\nu=1$ is the true static critical exponent rather than $\nu=$ 1.9 cannot be stated unambiguously from our data for $\theta<$ $1^{\circ}$ because such a fit is over a limited range and simply may be due to an intrinsic splay of heavy ion tracks about the crystal's $c$ axis.

[28] R. J. Olsson, W. K. Kwok, and G. W. Crabtree (unpublished).

[29] See, for example, M. V. Indenbom et al., Physica (Amsterdam) 226C, 325 (1994).

[30] E. Shimshoni, A. Auerbach, and A. Kapitulnik, Phys. Rev. Lett. 80, 3352 (1998). 